http://jmscr.igmpublication.org/home/ ISSN (e)-2347-176x ISSN (p) 2455-0450 crossref DOI: https://dx.doi.org/10.18535/jmscr/v9i1.21

\title{
Friedreich's Ataxia with Type I Diabetes and Cardiomyopathy in an Adolescent Girl- A Rare Case Study
}

\author{
Authors \\ Dr Upasana Patra ${ }^{1}$, Dr Chinmayee Mohanty ${ }^{2}$, Dr Budhia Majhi ${ }^{3}$, Dr Lal mohan Alada ${ }^{4}$ \\ ${ }^{1,2}$ Junior Resident, Dept of Paediatrics, MKCG Medical College, Berhampur, Odisha \\ ${ }^{3}$ Asso. Professor, Dept of paediatrics, MKCG Medical College, Berhampur, Odisha \\ ${ }^{4}$ Senior Resident, Dept of paediatrics, MKCG Medical College, Berhampur, Odisha
}

\begin{abstract}
Friedreich's ataxia is an autosomal recessive spinocerebellar ataxia involving neuromuscular and endocrine system. Usual presentation include progressive gait ataxia, absent deep tendon reflexes, extensor plantar response, cerebellar dysarthria, optic atrophy, neuropathy and loss of proprioception and vibration. Only few reports have documented the disease onset in childhood, so we are presenting a case of $13 y e a r$ female child with progressive unsteadiness of gait, increased urination, appetite, thirst which was ultimately diagnosed to have friedreich's ataxia with associated diabetes and cardiomyopathy. Currently, no definitive therapy is available. Screening, evaluation awareness of underlying endocrinopathies in friedreich's ataxia may provide new therapeutic targets for preventing Friedreich's ataxia associated cardiac dysfunction.
\end{abstract}

Keywords: Friedreich's ataxia; cerebellar dysarthria; cardiomyopathy; endocrinopathy; autosomal recessive.

\section{Introduction}

Friedreich's ataxia is one of the most common hereditary ataxia with prevalence of 1-2 per $1,00,000$ in the general population ${ }^{1}$. It is an autosomal recessive disorder due to trinucleotide repeat expansion of GAA in intron 1 of mitochondrial protein Frataxin gene located on chromosome 9. It was reported originally by Friedreich in 1863. Usual presentation is progressive gait ataxia, cerebellar dysarthria, neuropathy, distal wasting, optic atrophy, absent deep tendon reflexes, plantar extensor, loss of vibration and proprioception ${ }^{6}$. The natural history of this disease has a relentless progression.

\section{Case Study}

A 13 year old female child neurodevelopmentally normal, born of non consanguineous marriage from upper lower socioeconomic status presented to opd with chief complaints of unsteadiness of gait with swaying while walking which was gradually progressive over last 3 years, increased urination, increased thirst and appetite for 3 months. She had a history of skin eruption with fever and swelling 3yrs back following which unsteadiness in gait started. There was associated difficulty in wearing slippers, repeated occasional fall while playing in school, increase unsteadiness along narrow path and in dark. She was able to walk but took assistance from nearby objects and 
persons occasionally while walking at greater pace. There was no history of headache, vomiting, seizure, tingling, numbness, change in voice quality, nasal regurgitation, difficulty in deglutition, bladder and bowel incontinence. She had no difficulty in hearing or visual abnormality. She had no history of breathlessness, palpitation, chest pain, swelling of limbs. Family history of similar complaints were present in elder sister who had unsteady gait since childhood and was subsequently diagnosed to have diabetes in last 6 months and presently on insulin. O/E-child was conscious, oriented. HR-96/minute, RR-24/min, BP-110/70 mmHg, SPO2-100\% room air. Anthropometry showed wt-24kg, Ht-122cm, BMI- $16.7 \mathrm{~kg} / \mathrm{m} 2, \mathrm{Ht} / \mathrm{A}<3^{\text {rd }}$ percentile signifying short stature with undernutrition. There was presence of scoliosis and pes cavus (high arched feet) (fig 1) with no pallor, icterus, cyanosis, clubbing, lymphadenopathy. On systemic examination of cns- higher mental function was normal, cranial nerves were intact, bulk normal, tone diminished in all 4 limbs,power-5/5 in upper limb and $4+/ 5$ in lower limb, bilateral biceps, triceps and supinator jerks diminished with absent knee, ankle reflex, abdominal reflex intact with bilateral plantar extensor. She had wide based and ataxic gait. Vibration sensation diminished in all bony prominence with romberg's test positive. She had cerebellar signs like finger nose test, finger to finger nose test, dysdiadochokinesis, hypotonia, rebound phenomenon, knee heel test, tandem gait impaired, inability to walk in a straight line and all other systems being normal. On investigation- CBC normal, RBS high, ABGnormal, urine dipstick-glucose present, fundoscopy normal, MRI brain-normal (fig 2), MRI spine-mild thoracic scoliosis with convexity towards right side (fig 3), Nerve conduction velocities were normal, vitamin B12 normal, CXR-cardiomegaly with CT RATIO-0.6, 2DECHO-hypertrophic cardiomyopathy with high LV gradient, neurology consultation gave diagnosis as Friedreich's ataxia. Diagnosis of friedreich's ataxia was made on the basis of history, examination and few investigations as genetic confirmation facilities were not available. Subcutaneous insulin and physiotherapy was initiated. Parents were counselled about the disease and explained regarding possible outcome and necessity of follow up for timely evaluation of complications.

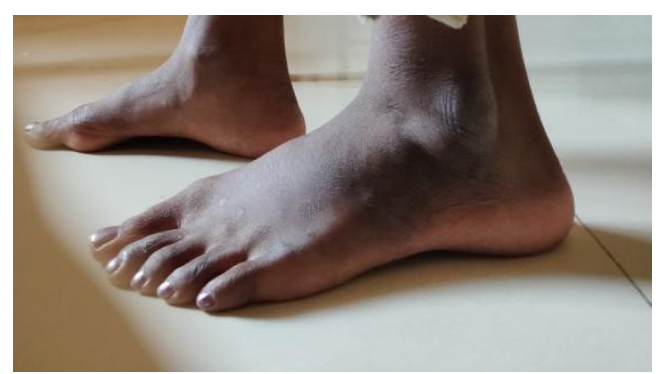

Fig 1

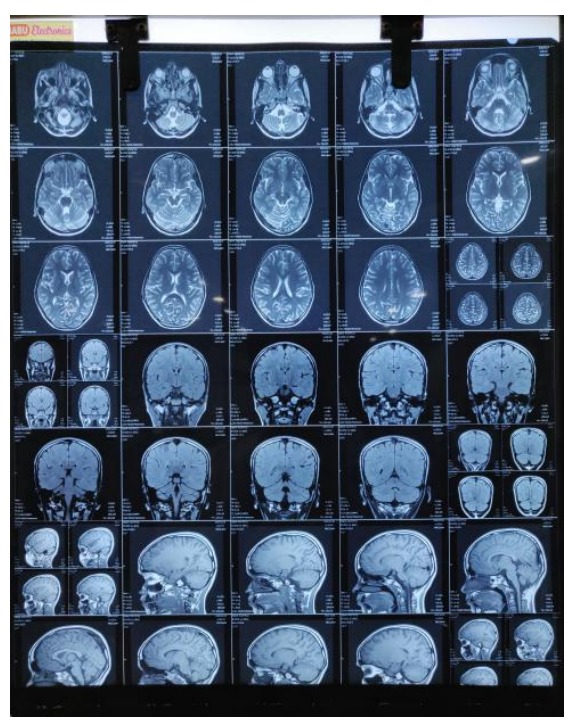

Fig 2

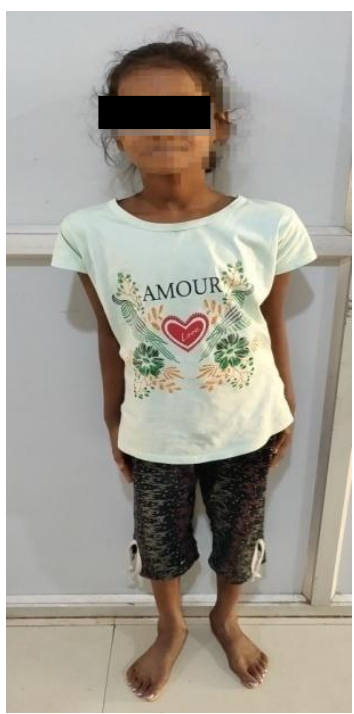

Fig 3 


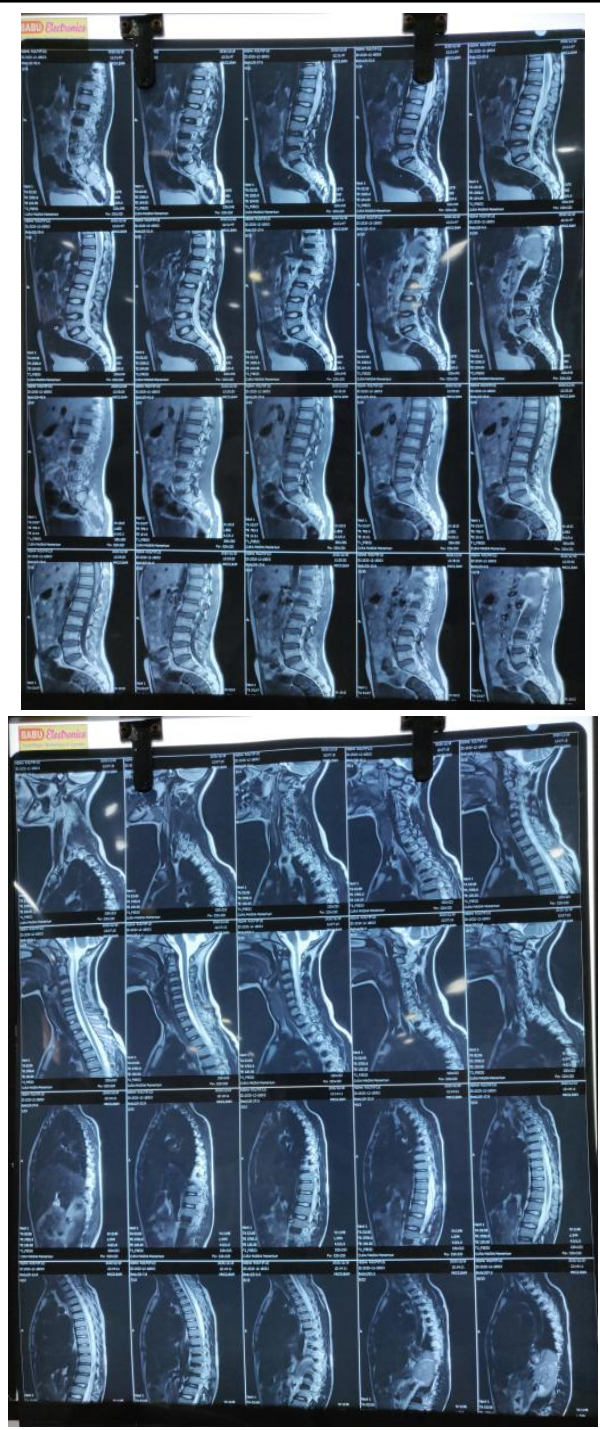

(MRI SPINE- Mild thoracic scoliosis with convexity towards right side (? Positional)

Lumbar vertebra and their appendages show normal narrow signal appearance. No altered signal intensity/ soft tissue replacement. No fracture. No displacement.)

\section{Discussion}

Friedreich's ataxia manifests usually before 10years in children or as late as third decade of life ${ }^{2}$.Typically a disease of young adults with no gender predilection ${ }^{5}$. It belongs to a family of rare neurodegenerative disorders known as autosomal recessive spinocerebellar ataxias. It affects cerebellum, medulla, spinocerebellar tract, pyramidal tract, posterior column in spinal cord and peripheral nerves. The first sign is usually progressive gait ataxia manifesting with widening of the base and wavering, associated with loss of proprioception ${ }^{1}$. Vibration and position sense grossly impaired with pain and temperature sensation lost in distal extremities. It is associated with areflexia, extensor plantar, cerebellar signs like nystagmus, dysarthria, truncal ataxia, intention tremors, choreiform movements over face and arms. Electrophysiologic study may show abnormalities in visual, auditory and somatosensory with fundus showing optic atrophy, retinitis pigmentosa etc ${ }^{1}$. Complex oculomotor disturbances are characteristic include abnormal saccades and square wave jerks. 60-79\% patients develop skeletal abnormalities like progressive scoliosis ${ }^{8}$ and others like high arched foot (pes cavus), hammer toes. Deep tendon reflexes (ankle reflex mainly) absent with bilateral plantar extensor. Approximately twenty percent of patients show abnormal glucose tolerance and another $10 \%$ develop diabetes mellitus due to beta cell failure and insulin resistance ${ }^{7}$. Diabetes mellitus due to FA is associated with higher ophthalmological complications ${ }^{3}$. There was no such complication in our patient. Most common cause of death in FA is cardiac failure due to cardiomyopathy and arrhythmias at middle thirties constituting around two third of patients. The incidence and progression of cardiomyopathy is directly proportional to the length of GAA expansion ${ }^{4}$. The rapid progression of cardiac symptoms in FA is suspected to be due to decreased level of frataxin leading to increased sensitivity to oxidative stress in cardiac tissues as a result of mitochondrial dysfunction. MRI spine shows atrophy of cervical spinal cord and atrophy of superior cerebellar peduncles. Nerve conduction velocity is diminished. Confirmation through genetic diagnosis is most sensitive and specific. No definitive treatment is available yet. Antioxidants like vit E/CoQ10 have proven effective. Idebenone, a short chain analog of CoQ 10, improve cardiac function and decrease ventricular mass ${ }^{1}$.

\section{Conclusion}

Diagnosis of Friedreich's ataxia usually begins with detailed history of onset of symptoms and 
assessment of family history followed by physical examination. Prognosis of Friedreich's ataxia is poor. Regular follow up with appropriate investigations is vital to detect associated complications early. No clear relationship is found between FRATAXIN mutation and cardiac dysfunction. We conclude that further investigation of endocrinopathies in Friedreich's ataxia may provide novel targets for preventing severe FA-associated cardiomyopathy.

\section{References}

1. Swaiman f.kenneth, Ashwal Stephen, ferriro $m$ donna, pediatric neurology, principles and practice; cerebellum and the hereditary ataxia, $6^{\text {th }}$ edition; 689-94.

2. Nelson textbook of paediatrics, $21^{\text {st }}$ edition: ataxia: 3150-54

3. Harding AE. Friedreich ataxia: A clinical and genetic study of 90 families with an analysis of early diagnostic criteria and intrafamilial clustering of clinical features. Brain 1981;104(3):589-620.

4. Filla A, De Michele G, Cavalcanti F, Pianese L, Monticelle A, Campanella G. The relationship between trinucleotide (GAA) repeat length and clinical features in Friedreich's ataxia. Am J Hum Genet 1996; 59(3):559-560.

5. Koeppen AH .F riedreich's ataxia: Pathology, pathogenesis and molecular genetics. J Neurol Sci.2011;303:1-29

6. Geoffroy G, Barbeau A ,Breton G, Lemieux B, Aube M, Leger C, et al. Clinical description and roentgenologic evaluation of patients with Friedreich's ataxia. Can J Neurol Sci 1976;3;279-86

7. Pandolfo, M. Friedreich ataxia: The clinical picture. J Neurol.2009;256 (suppl 1):3-8.google scholar
8. Weidemann F, Rummey C, Bijnens B, Stork S, Jasaityte R, Dhooge J, et al. The Heart in Friedreich Ataxia: Definition of cardiomyopathy, disease severity, and correlation with neurological symptoms. Circulation 2012;125(13):1626-34. 